\title{
Robustness of the neurological prognostic score in brain metastasis patients treated with Gamma Knife radiosurgery
}

\author{
Toru Serizawa, MD, ${ }^{1}$ Yoshinori Higuchi, MD, ${ }^{2}$ Osamu Nagano, MD, ${ }^{3}$ Shinji Matsuda, MD, ${ }^{4}$ \\ Kyoko Aoyagi, MD, ${ }^{3}$ Junichi Ono, MD, ${ }^{3}$ Naokatsu Saeki, MD, ${ }^{2}$ Yasuo Iwadate, MD, ${ }^{2}$ \\ Tatsuo Hirai, MD, ${ }^{5}$ Shinya Takemoto, MD, ${ }^{6}$ and Yuta Shibamoto, MD ${ }^{6}$
}

\begin{abstract}
${ }^{1}$ Tokyo Gamma Unit Center, Tsukiji Neurological Clinic, Tokyo; ${ }^{2}$ Department of Neurological Surgery, Chiba University Graduate School of Medicine, Chiba; ${ }^{3}$ Gamma Knife House, Chiba Cerebral and Cardiovascular Center, Ichihara; ${ }^{4}$ Department of Neurology and Strokology, Chiba Central Medical Center, Chiba; ${ }^{5}$ Heisei Gamma Unit Center, Fujieda Heisei Memorial Hospital, Fujieda; and ${ }^{6}$ Department of Radiology, Graduate School of Medical Sciences and Medical School, Nagoya City University, Nagoya, Japan
\end{abstract}

\begin{abstract}
OBJECTIVE The neurological prognostic score (NPS) was recently proposed as a means for predicting neurological outcomes, such as the preservation of neurological function and the prevention of neurological death, in brain metastasis patients treated with Gamma Knife radiosurgery (GKRS). NPS consists of 2 groups: Group A patients were expected to have better neurological outcomes, and Group B patients were expected to have poorer outcomes. NPS robustness was tested in various situations.
\end{abstract}

METHODS In total, 3040 patients with brain metastases that were treated with GKRS were analyzed. The cumulative incidence of the loss of neurological function independence (i.e., neurological deterioration) was estimated using competing risk analysis, and NPS was compared between Groups A and B by employing Gray's model. NPS was tested to determine if it can be applied to 5 cancer categories-non-small cell lung cancer, small cell lung cancer, gastrointestinal tract cancer, breast cancer, and other cancers-as well as if it can be incorporated into the 5 major grading systems: recursive partitioning analysis (RPA), score index for stereotactic radiosurgery (SIR), basic score for brain metastases (BSBM), graded prognostic assessment (GPA), and modified-RPA (M-RPA).

RESULTS There were 2263 patients in NPS Group A and 777 patients in Group B. Neurological deterioration was observed in 586 patients (19.2\%). The cumulative incidences of neurological deterioration were $9.5 \%$ versus $21.0 \%$, $14.1 \%$ versus $25.4 \%$, and $17.6 \%$ versus $27.8 \%$ in NPS Groups A and B at 1,2 , and 5 years, respectively. Significant differences were detected between the NPS groups in all cancer categories. There were significant differences between NPS Groups A and B for all classes in terms of the BSBM, GPA, and M-RPA systems, but the differences failed to reach statistical significance in terms of RPA Class I and SIR Class 0 to 3.

CONCLUSIONS The NPS was verified as being highly applicable to all cancer categories and almost all classes for the 5 grading systems in terms of neurological function independence. This NPS system appears to be quite robust in various situations for brain metastasis patients treated with GKRS.

https://thejns.org/doi/abs/10.3171/2016.8.JNS16528

KEY WORDS neurological prognostic score; neurological function independence; neurological deterioration; qualitative survival; Gamma Knife radiosurgery; brain metastases; oncology; stereotactic radiosurgery

$\mathrm{N}$ EUROLOGICAL outcomes, such as the preservation of neurological function (qualitative survival) and the prevention of neurological death (neurological survival), are regarded as the ideal end points for evaluating the treatment results of patients with brain metastases, as well as overall survival. ${ }^{8,9}$ In a previous study, we proposed using the neurological prognostic score (NPS) as a means of predicting both qualitative and neurological survival in brain metastasis patients treated with Gamma Knife radiosurgery (GKRS). ${ }^{9}$ This NPS system consists of 4 intracra-

ABBREVIATIONS BSBM = basic score for brain metastases; CITV = cumulative intracranial tumor volume; DS-GPA = diagnosis-specific graded prognostic assessment; GKRS = Gamma Knife radiosurgery; GPA = graded prognostic assessment; KPS = Karnofsky Performance Scale; M-RPA = modified recursive partitioning analysis; NPS = neurological prognostic score; RPA = recursive partitioning analysis; SIR = score index for stereotactic radiosurgery.

SUBMITTED March 1, 2016. ACCEPTED August 10, 2016.

INCLUDE WHEN CITING Published online December 2, 2016; DOI: 10.3171/2016.8.JNS16528. 
nial disease factors: brain tumor number $(\leq 10$ vs $>10)$, cumulative intracranial tumor volume (CITV) $\left(\leq 15 \mathrm{~cm}^{3}\right.$ vs $>15 \mathrm{~cm}^{3}$ ), MRI findings of localized leptomeningeal dissemination (no vs yes), and neurological symptoms (no vs yes). For each of these intracranial disease factors, a score of 0 indicates a poor prognosis, and a score of 1 indicates a good neurological prognosis. According to the sum of these NPS values, we classified Group A as having a score of 3 or 4 and Group B as a score of 0,1 , or 2 . Thus, better neurological outcomes can be expected in NPS Group A and poorer outcomes in Group B. In this study, we tested the robustness of this NPS system in various situations with a focus on neurological function independence.

\section{Methods}

A total of 3040 consecutive cases that were treated with GKRS by the first author (T.S.) were analyzed. The database consisted of 2 cohorts that were studied retrospectively after institutional review board approval: the Chiba series (1716 patients treated between January 1998 and March 2008) plus the Tokyo series (1324 patients treated between April 2008 and December 2014). During this 17 -year period, all patients were treated according to the same indications and protocol, as reported previously. ${ }^{10,12}$ Our major inclusion criteria for GKRS are a tumor number up to 25 , maximum tumor diameter less than $4 \mathrm{~cm}$, no MRI findings of diffuse leptomeningeal dissemination, and a Karnofsky Performance Scale (KPS) score of 70 or better. However, we applied these indications with flexibility, especially for patients with special circumstances such as contraindications for whole-brain radiotherapy and craniotomy or the expectation of a good response to small molecular targeting agents. ${ }^{6}$ Patients with KPS scores less than 70 due to focal neurological signs and symptoms and whose performance status was expected to improve after GKRS were included. Furthermore, patients with functional independence before their cancer diagnosis-e.g., patients with cerebral palsy, polio infection, or spinal cord injuries-were also considered to be suitable candidates for GKRS. None of the cases in this series had diffuse leptomeningeal dissemination, which is widely regarded as a contraindication for GKRS. Leptomeningeal dissemination always indicates localized disease, meaning that the tumor margin is in contact with cerebrospinal fluid spaces. Our GKRS-alone treatment protocol is as follows: large tumors $(>3 \mathrm{~cm}$ in diameter) were surgically removed or irradiated with staged GKRS, 3,17 and all other smaller tumors were irradiated with GKRS. We do not perform upfront whole-brain radiotherapy. New distant lesions, as detected with follow-up MRI, were appropriately irradiated with GKRS.

In this study, we focused on neurological functional independence. We defined functional independence as a KPS score of 70 or better. We divided the loss of functional independence into "neurological deterioration" due to intracranial disease progression and "systemic deterioration" due to extracranial disease progression. Thus, neurological deterioration was defined as a KPS score that decreased to less than 70 due to intracranial disease progression. ${ }^{9-11}$ The interval from the date of GKRS until the date of loss of functional independence was calculated. We categorized functional independence as "censored," neurological deterioration as "failure," and systemic deterioration as a "competing risk." For patients with an initial KPS score lower than 70 and who never achieved a KPS score of at least 70 after GKRS, we substituted the surrogate end point of a 20-point decrease in KPS score as a representation of functional dependence. Thus, we estimated the cumulative incidence of neurological deterioration by employing the competing risk analysis model. ${ }^{2}$ We divided the cases into 5 cancer categories: non-small cell lung cancer, small cell lung cancer, gastrointestinal tract cancer, breast cancer, and other cancers. We also examined 5 major grading systems: recursive partitioning analysis (RPA), ${ }^{1}$ score index for stereotactic radiosurgery (SIR), ${ }^{15}$ basic score for brain metastases (BSBM), ${ }^{5}$ graded prognostic assessment (GPA), ${ }^{13}$ and modified RPA (M-RPA). ${ }^{16}$ The cumulative neurological deterioration incidences were compared between the 2 NPS groups and among the 5 cancer categories and 5 major grading systems by employing Gray's test. ${ }^{7}$ A p value $<0.05$ was considered to be statistically significant. All statistical analyses were performed using EZR (Saitama Medical Center, Jichii Medical University), which is a graphical user interface for $\mathrm{R}$ (The $\mathrm{R}$ Foundation for Statistical Computing). ${ }^{4}$ More precisely, it is a modified version of the $\mathrm{R}$ commander that is designed to add statistical functions frequently used in biostatistics. ${ }^{4}$

\section{Results}

Table 1 summarizes the patient backgrounds. At the end of December 2015, 222 (7.3\%) patients were still alive and the remaining $2818(92.7 \%)$ patients were confirmed to have died. The median survival time was 8.0 months. Among 2829 patients with loss of function independence, neurological deterioration was observed in 593 patients and systemic deterioration was observed in 2236 patients. Figure 1 shows the cumulative incidences of systemic and neurological deterioration. The cumulative incidences of loss of function independence were $65.6 \%$ (12.4\% for neurological deterioration vs $53.4 \%$ for systemic deterioration), $83.2 \%$ ( $17.0 \%$ vs $66.2 \%$ ), $90.4 \%$ ( $18.6 \%$ vs $71.8 \%$ ), $93.9 \%$ (19.6\% vs $74.3 \%$ ), and $95.7 \%$ (20.2\% vs $75.5 \%)$ at $1,2,3,4$, and 5 years, respectively. The causes of neurological deterioration were leptomeningeal dissemination progression in 184 patients $(31.2 \%)$, GKRS-treated lesion regrowth in 140 (23.6\%) patients, cerebral dissemination in 51 (8.6\%) patients, the growth of untreated lesions in $46(7.6 \%)$ patients, and unknown or other causes in $172(29.0 \%)$ patients. The crude incidences of neurological deterioration were $16.7 \%$ (378 patients) in NPS Group A and 26.8\% (208 patients) in Group B. Figure 2 shows neurological deterioration, which allowed the NPS groups to be compared. The cumulative neurological deterioration incidences were $9.5 \%$ versus $21.0 \%, 14.1 \%$ versus $25.4 \%, 15.9 \%$ versus $26.3 \%, 17.1 \%$ versus $27.0 \%$, and $17.6 \%$ versus $27.8 \%$ in NPS Groups A and $\mathrm{B}$ at $1,2,3,4$, and 5 years, respectively. The differences were highly significant $(\mathrm{p}<0.0001)$.

Figure 3 shows neurological deterioration, which allowed comparison of the NPS groups for each of the 5 cancer categories. There were significant differences between 
TABLE 1. Characteristics of 3040 brain metastasis patients

\begin{tabular}{|c|c|}
\hline Variable & Value* $^{*}$ \\
\hline \multicolumn{2}{|l|}{ Age } \\
\hline Median (IQR), yrs & $65(58-72)$ \\
\hline$\geq 65$ & $1606(52.3)$ \\
\hline$<65$ & $1434(47.1)$ \\
\hline \multicolumn{2}{|l|}{ Sex } \\
\hline Male & $1798(59.1)$ \\
\hline Female & $1242(40.9)$ \\
\hline \multicolumn{2}{|l|}{ KPS score } \\
\hline $80-100$ & $2327(76.5)$ \\
\hline $50-70$ & $713(23.5)$ \\
\hline \multicolumn{2}{|l|}{ Controlled primary cancer } \\
\hline Yes & $948(31.2)$ \\
\hline No & $2092(68.8)$ \\
\hline \multicolumn{2}{|l|}{ Extracranial metastasis } \\
\hline Yes & $1951(64.2)$ \\
\hline No & $1089(35.8)$ \\
\hline \multicolumn{2}{|l|}{ Primary cancer } \\
\hline Non-small cell lung & $1730(56.9)$ \\
\hline Small cell lung & $282(9.3)$ \\
\hline Gastrointestinal tract & $375(12.4)$ \\
\hline Breast & $329(10.8)$ \\
\hline Other & $324(10.7)$ \\
\hline \multicolumn{2}{|c|}{$\begin{array}{l}\text { Diagnostic lag btwn primary cancer \& } \\
\text { brain metastases }\end{array}$} \\
\hline Synchronous & $1074(35.3)$ \\
\hline Metachronous & $1966(64.7)$ \\
\hline Prior craniotomy & $404(13.3)$ \\
\hline Prior whole-brain radiotherapy & $233(7.7)$ \\
\hline \multicolumn{2}{|l|}{ No. of brain tumors } \\
\hline Median (IQR) & $3(1-7)$ \\
\hline Single & $855(28.1)$ \\
\hline $2-4$ & $1001(32.9)$ \\
\hline $5-10$ & $667(21.9)$ \\
\hline$>10$ & $517(17.0)$ \\
\hline \multicolumn{2}{|l|}{ Maximum tumor diameter } \\
\hline Median (IQR), mm & $20.8(13.5-29.5)$ \\
\hline$\geq 25 \mathrm{~mm}$ & $1106(36.4)$ \\
\hline$<25 \mathrm{~mm}$ & $1934(63.6)$ \\
\hline \multicolumn{2}{|l|}{ Cumulative intracranial tumor vol } \\
\hline Median (IQR), $\mathrm{cm}^{3}$ & $4.4(1.4-11.1)$ \\
\hline$>15$ & $545(17.9)$ \\
\hline$\leq 15$ & $2495(82.1)$ \\
\hline \multicolumn{2}{|c|}{$\begin{array}{l}\text { MRI findings of localized leptomenin- } \\
\text { geal dissemination }\end{array}$} \\
\hline Yes & $331(10.9)$ \\
\hline No & $2709(89.1)$ \\
\hline \multicolumn{2}{|l|}{ Neurological symptoms } \\
\hline Yes & $1446(47.6)$ \\
\hline No & $1594(52.4)$ \\
\hline
\end{tabular}

NPS Groups A and B in all cancer categories $(\mathrm{p}=0.0002$ for non-small cell lung cancer, $p=0.0235$ for small cell lung cancer, $p=0.0439$ for gastrointestinal tract cancer, $p=$ 0.0042 for breast cancer, and $p=0.0011$ for other cancers).

Table 2 summarizes the comparisons of the cumulative neurological deterioration incidences between NPS Groups A and B for each class of RPA, SIR, BSBM, GPA, and M-RPA. For RPA, there were significant differences in Class II $(n=2484 ; \mathrm{p}<0.0001)$ and Class III $(\mathrm{n}=384$; $\mathrm{p}=0.0010)$, but not Class I $(\mathrm{n}=172 ; \mathrm{p}=0.342)$. For SIR, there were significant differences in SIR Class 4 to 7 (n $=2340 ; \mathrm{p}<0.0001)$ and SIR Class 8 to $10(\mathrm{n}=253$; $\mathrm{p}<$ $0.0001)$, but not SIR Class 0 to $3(n=347 ; p=0.0577)$. For BSBM, there were significant differences in all classes. The $\mathrm{p}$ values were $<0.0001$ for BSBM Class $0(\mathrm{n}=402)$, $<0.0001$ for BSBM Class $1(\mathrm{n}=1243), 0.0004$ for BSBM Class $2(n=1064)$, and 0.0002 for BSBM Class $3(n=330)$. For GPA, there were significant differences in all classes. The $\mathrm{p}$ values were 0.0001 for GPA Class 0 to $1(\mathrm{n}=1288)$, $<0.0001$ for GPA Class 1.5 to $2.5(\mathrm{n}=1535), 0.0002$ for GPA Class $3.0(n=163)$, and 0.0213 for GPA Class 3.5 to $4.0(n=49)$. For M-RPA, there were significant differences in all classes. The $p$ values were $p<0.0001$ for M-RPA Class I+IIa ( $\mathrm{n}=564), 0.0014$ for M-RPA Class IIb ( $\mathrm{n}=$ $871)$, and $<0.0001$ for M-RPA Class IIc+III $(n=1605)$.

\section{Discussion}

We proposed using the NPS system for brain metastasis patients treated with GKRS as a means of predicting neurological outcomes such as qualitative and neurological survival. ${ }^{9}$ NPS consists of 4 intracranial disease factors: brain tumor number, CITV, an MRI finding of localized leptomeningeal dissemination, and neurological symptoms. We classified NPS Group A as those patients for which better neurological outcomes could be expected and Group B as those patients with poorer anticipated outcomes. The 5 major grading systems-RPA, SIR, BSBM, GPA, and M-RPA-can predict life expectancy but not neurological outcomes. Our NPS is unique because this is the only system that can predict neurological outcomes, the ideal end point for evaluating GKRS, as well as other radiation treatment results for patients with brain metastases. Furthermore, NPS can be incorporated into the 5 major grading systems, such that we can predict life expectancy and neurological outcomes independently.

In our previous study, we estimated qualitative and neurological survival by employing the Kaplan-Meier method. ${ }^{9}$ However, these cumulative incidences tended to be overestimates compared with the true values because the Kaplan-Meier method does not take competing risk into consideration. ${ }^{2}$ For example, neurological deterioration cannot be observed if patients experience systemic deterioration and vice versa. Neurological and systemic deterioration are considered competing factors for each other. In this study, we applied competing risk analysis and estimated much more accurately the cumulative incidences of neurological deterioration. F Furthermore, we tested if our NPS system can be applied to 5 primary cancer sites, as well as if it can be incorporated into 5 grading systems, in terms of evaluating neurological deterioration using Gray's test. 


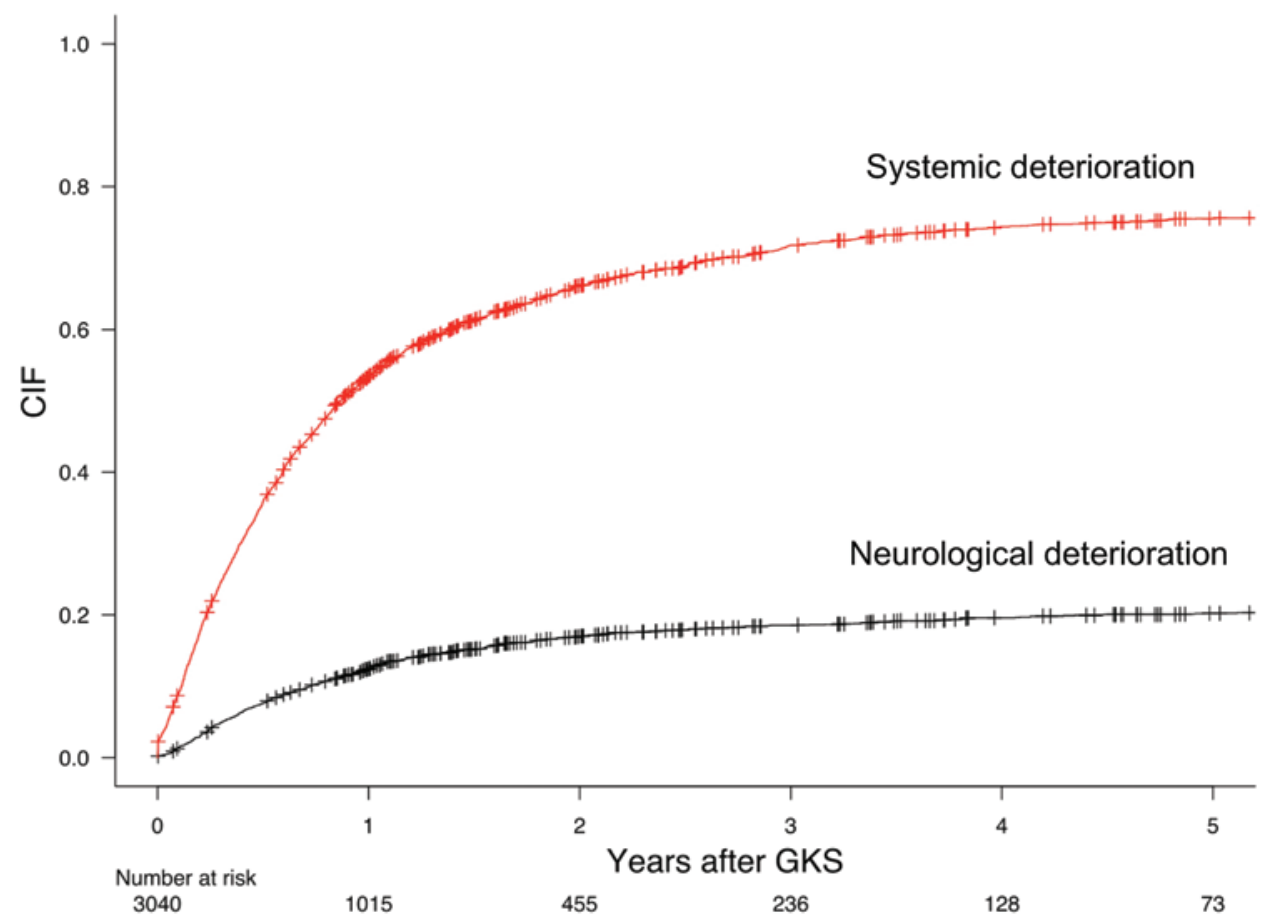

FIG. 1. Cumulative incidences of the loss of function independence due to systemic disease progression and neurological disease progression. The red line indicates systemic deterioration, and the black line indicates neurological deterioration. The cumulative incidences of the loss of function independence were $65.6 \%$ (12.4\% for neurological deterioration vs $53.4 \%$ for systemic deterioration), $83.2 \%$ (17.0\% vs $66.2 \%$ ), $90.4 \%$ (18.6\% vs $71.8 \%$ ), $93.9 \%$ (19.6\% vs $74.3 \%$ ), and $95.7 \%(20.2 \%$ vs $75.5 \%)$ at 1,2 , 3,4 , and 5 years, respectively. CIF = cumulative incidence function; GKS = Gamma Knife (radio)surgery. Figure is available in color online only.

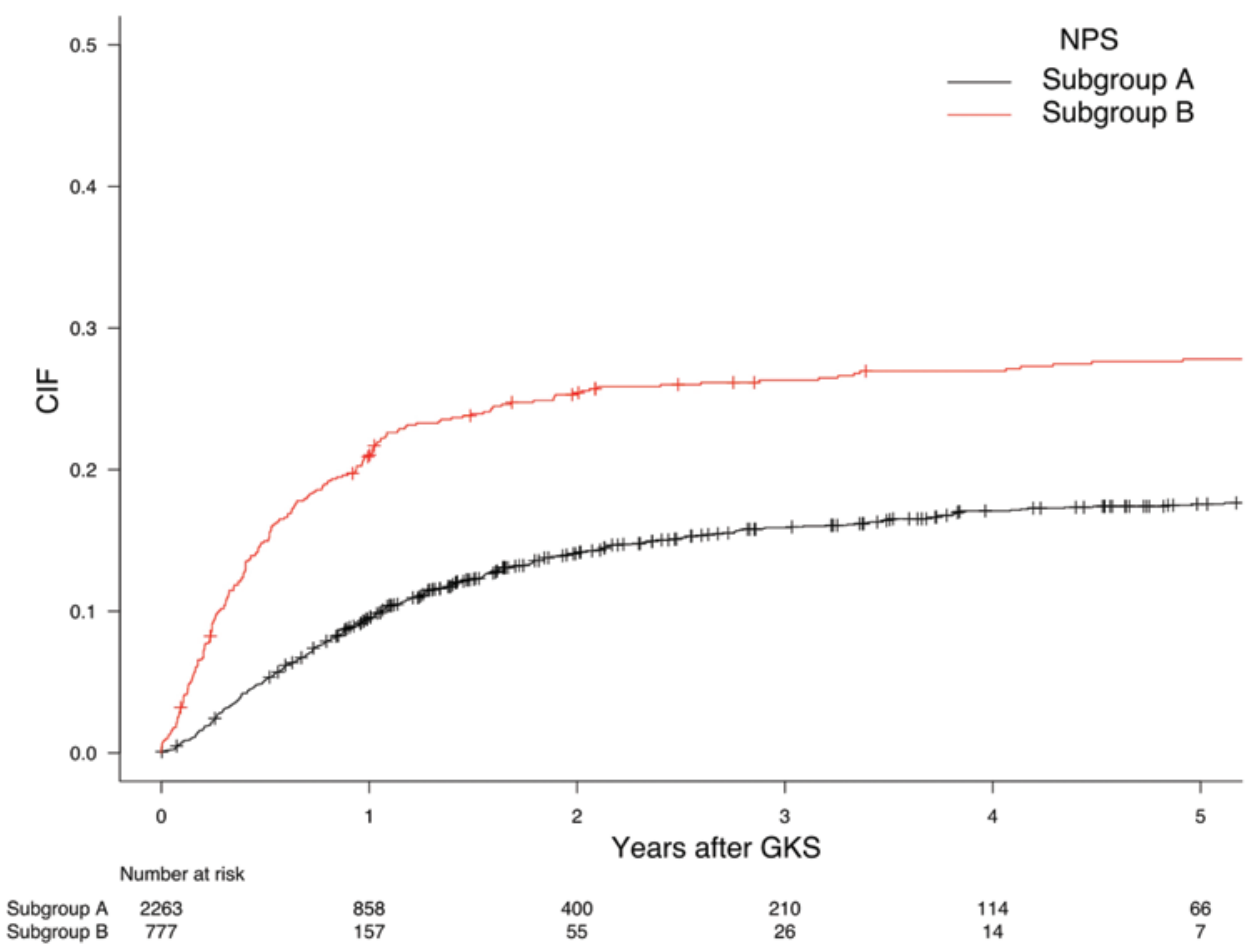

FIG. 2. Comparison of neurological deterioration between NPS Groups $A$ and $B$. The black line indicates NPS Group A, and the red line indicates Group B. The cumulative incidences of neurological deterioration were $9.5 \%$ versus $21.0 \%, 14.1 \%$ versus $25.4 \%$, $15.9 \%$ versus $26.3 \%, 17.1 \%$ versus $27.0 \%$, and $17.6 \%$ versus $27.8 \%$ in NPS Groups A and B at $1,2,3,4$, and 5 years, respectively. The difference between the 2 NPS groups was highly significant $(p<0.0001)$. Figure is available in color online only. 

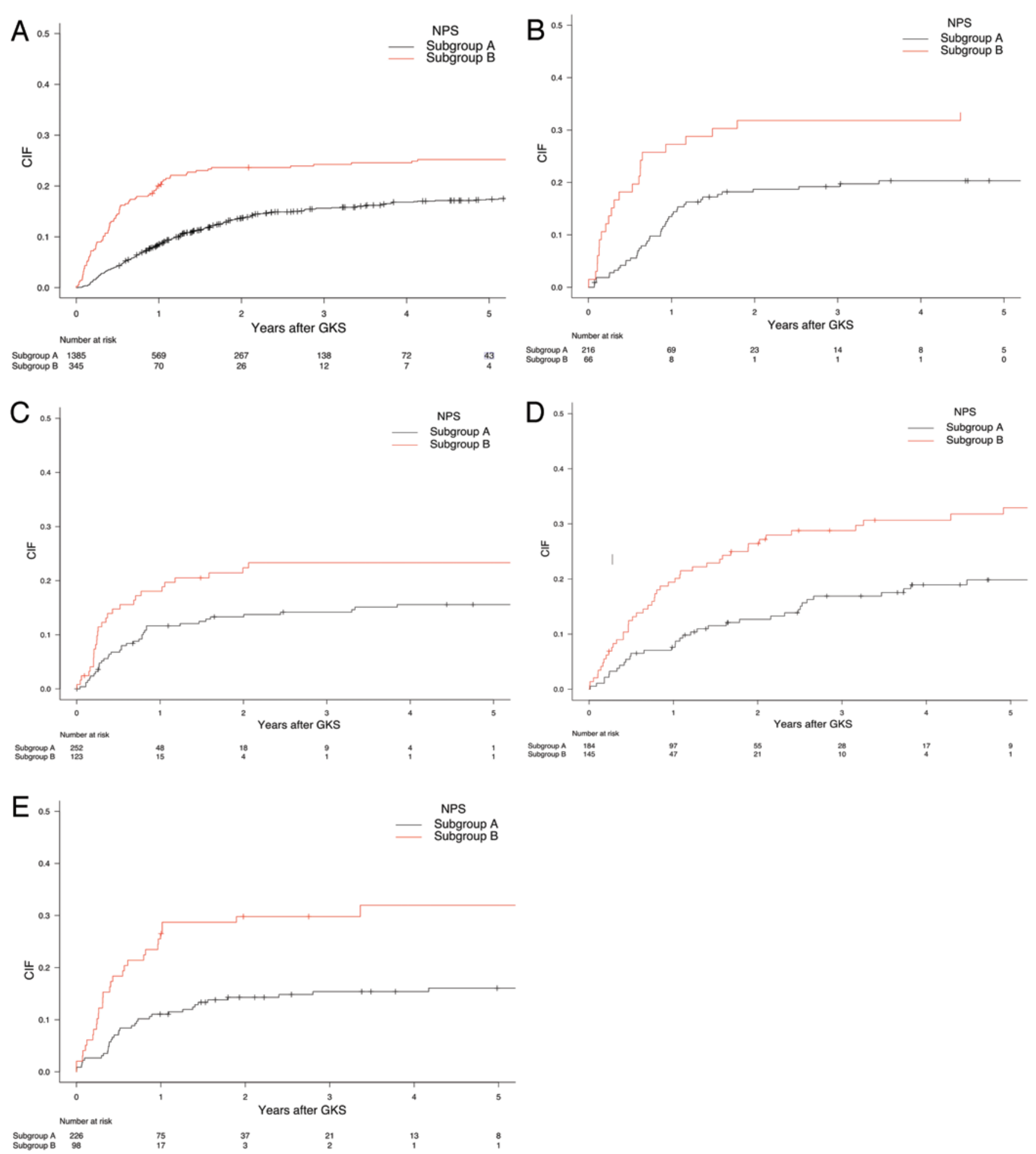

FIG. 3. Cumulative incidences of neurological deterioration that allow comparison between the NPS groups for the 5 cancer categories. A: Non-small cell lung cancer. B: Small cell lung cancer. C: Gastrointestinal cancer. D: Breast cancer. E: Other cancers. The black line indicates NPS Group A, and the red line indicates Group B. There were significant differences between the 2 NPS groups for each cancer category $(p=0.0002$ for non-small cell lung cancer, $p=0.0235$ for small cell lung cancer, $p=0.0439$ for gastrointestinal cancer, $p=0.0042$ for breast cancer, and $p=0.0011$ for other cancers). Figure is available in color online only.

First, we ascertained the validity of our NPS system according to 5 primary cancer types-non-small cell lung cancer, small cell lung cancer, gastrointestinal cancer, breast cancer, and other cancers-and found statistically significant differences between NPS Groups A and B for all 5 categories. Thus, the NPS system was applicable to 
TABLE 2. Comparison of the cumulative neurological deterioration incidences between NPS Groups A and B according to 5 major grading systems

\begin{tabular}{|c|c|c|c|c|}
\hline Grading System & $1 \mathrm{Yr}$ & 2 Yrs & 5 Yrs & $p$ Value \\
\hline \multicolumn{5}{|l|}{ RPA } \\
\hline Class I & & & & 0.3420 \\
\hline Group A & 10.1 & 21.3 & 31.1 & \\
\hline Group B & 18.2 & 28.0 & 37.8 & \\
\hline Class II & & & & $<0.0001$ \\
\hline Group A & 9.7 & 13.7 & 17.1 & \\
\hline Group B & 20.8 & 25.8 & 28.4 & \\
\hline Class III & & & & 0.0010 \\
\hline Group A & 10.7 & 11.8 & 11.8 & \\
\hline Group B & 22.2 & 23.9 & NA & \\
\hline \multicolumn{5}{|l|}{ SIR } \\
\hline Class 0-3 & & & & 0.0577 \\
\hline Group A & 11.9 & 14.3 & NA & \\
\hline Group B & 20.0 & 21.2 & $\mathrm{BA}$ & \\
\hline Class 4-7 & & & & $<0.0001$ \\
\hline Group A & 9.6 & 14.3 & 17.6 & \\
\hline Group B & 21.6 & 27.1 & 30.2 & \\
\hline Class 8-10 & & & & $<0.0001$ \\
\hline Group A & 6.3 & 12.3 & 19.9 & \\
\hline Group B & 23.1 & 53.8 & 61.5 & \\
\hline \multicolumn{5}{|l|}{ BSBM } \\
\hline Class 0 & & & & 0.0031 \\
\hline Group A & 9.6 & 9.6 & NA & \\
\hline Group B & 20.3 & 20.3 & 21.9 & \\
\hline Class 1 & & & & $<0.0001$ \\
\hline Group A & 9.4 & 12.2 & 12.9 & \\
\hline Group B & 20.9 & 25.1 & NA & \\
\hline Class 2 & & & & 0.0004 \\
\hline Group A & 9.8 & 15.7 & 19.4 & \\
\hline Group B & 19.7 & 26.9 & 30.5 & \\
\hline Class 3 & & & & 0.0002 \\
\hline Group A & 8.8 & 18.4 & 30.8 & \\
\hline Group B & 34.3 & 45.7 & 57.1 & \\
\hline \multicolumn{5}{|l|}{ GPA } \\
\hline Class $0-1.0$ & & & & 0.0001 \\
\hline Group A & 11.2 & 14.2 & 16.0 & \\
\hline Group B & 19.7 & 22.8 & 23.9 & \\
\hline Class $1.5-2.5$ & & & & $<0.0001$ \\
\hline Group A & 8.8 & 13.9 & 17.5 & \\
\hline Group B & 22.6 & 29.1 & 33.3 & \\
\hline Class 3.0 & & & & 0.0002 \\
\hline Group A & 8.6 & 15.6 & 24.3 & \\
\hline Group B & 45.5 & 56.8 & 68.2 & \\
\hline Class $3.5-4.0$ & & & & 0.0213 \\
\hline Group A & 2.3 & 11.7 & 22.4 & \\
\hline Group B & 40.0 & 40.0 & 60.0 & \\
\hline
\end{tabular}

" CONTINUED FROM PREVIOUS COLUMN

TABLE 2. Comparison of the cumulative neurological deterioration incidences between NPS Groups A and B according to 5 major grading systems

\begin{tabular}{crrrr}
\hline Grading System & 1 Yr & 2 Yrs & 5 Yrs & p Value \\
\hline M-RPA & & & & $<0.0001$ \\
\hline Class I+Ila & & & & $<0.0001$ \\
\hline Group A & 7.4 & 14.8 & 23.9 & \\
\hline Group B & 29.4 & 41.4 & 50.8 & \\
\hline Class Ilb & & & & 0.0014 \\
\hline Group A & 10.4 & 16.3 & 19.6 & \\
\hline Group B & 20.6 & 27.9 & 31.3 & \\
\hline Class IIc+III & & & & $<0.0001$ \\
\hline Group A & 10.0 & 12.2 & 12.9 & \\
\hline Group B & 20.4 & 23.4 & 25.1 & \\
\hline
\end{tabular}

$\mathrm{NA}=$ not available.

Values are shown as percentages unless otherwise indicated.

all primary cancer types in our series. Next, we also tested the validity of the NPS system in each class of 5 grading systems: RPA, ${ }^{1}$ SIR, ${ }^{15}$ BSBM,${ }^{5}$ GPA,${ }^{13}$ and M-RPA. ${ }^{16}$ These grading systems are highly applicable to patients treated with GKRS in terms of overall survival, as reported previously. ${ }^{8}$ We detected statistically significant differences in almost all classes of the aforementioned 5 grading systems. In RPA Class I and SIR Class 0 to 3, however, the differences did not reach statistical significance. We assume that this was due to the number of RPA Class I cases being too small $(n=172)$. SIR has 2 brain items (maximum tumor volume and tumor number) that are used for classification and can thus predict neurological outcomes such as qualitative survival, as reported previously. ${ }^{8}$ The NPS system has similar items: CITV and tumor number. For SIR, the number of tumors was classified as 1,2 , or $\geq 3$, whereas NPS uses $\leq 10$ and $>10$. We assume that maximum tumor volume correlates very strongly with CITV. This overlapping may account for the lack of statistically significant differences in SIR Class 0 to $3(\mathrm{p}=0.0577)$ despite the number of SIR Class 0 to 3 cases being relatively large (n =348). Although GPA and M-RPA also use 1 brain factor (tumor number, which is classified as single or multiple) and the number of GPA cases (Class 3.0 and 3.5-4.0) was very small, there were highly statistically significant differences in all classes. Thus, our NPS system proved to be extremely robust in various situations.

We have highlighted the usefulness of incorporating NPS into established grading systems because this will provide very important information for selecting the optimal radiation treatment intensity when performing GKRS. ${ }^{9}$ What is the best grading system into which NPS can be incorporated? For statistical reasons, RPA and SIR are not recommended. Considering the overlapping of the evaluated items, GPA and M-RPA were not ideal. BSBM would thus be best. However, we found highly significant differences between NPS Groups A and B in all GPA and M-RPA classes. We conclude that BSBM, GPA, and M-RPA are the best grading systems for incorporation into NPS. Recently, Sperduto et al. modified their original 
GPA system and developed a new index: diagnosis-specific GPA (DS-GPA). ${ }^{14}$ We plan to perform validity testing to determine if NPS can be incorporated into DS-GPA, but the number of cases in each class is still too small.

It should be noted that our NPS system has limitations. This was a two-institution, retrospective study. Furthermore, 2838 patients $(93.4 \%)$ in the original NPS system report were among the 3040 patients included in this study. Validity testing on the other groups using patient selection criteria and practice patterns that are different from our series, especially those conducted outside of Japan, is needed. Clinical trials that assess the usefulness of our NPS system when incorporated into grading systems for predicting overall survival are also necessary. Finally, our NPS system can only be used at the completion of dose planning for radiosurgery because NPS scores can change between clinical diagnosis and GKRS due to an increase in the number of tumors, changes in neurological symptoms, the emergence of leptomeningeal dissemination, and/or increased CITV. However, our NPS system can predict neurological outcomes such as the deterioration of neurological function at the time of radiosurgery and provide important information as to whether GKRS alone would be sufficient treatment.

\section{Conclusions}

Our NPS system can predict the preservation of neurological function independence and was found to be extremely robust when applied to brain metastasis patients who were treated with GKRS in various situations.

\section{References}

1. Gaspar L, Scott C, Rotman M, Asbell S, Phillips T, Wasserman T, et al: Recursive partitioning analysis (RPA) of prognostic factors in three Radiation Therapy Oncology Group (RTOG) brain metastases trials. Int J Radiat Oncol Biol Phys 37:745-751, 1997

2. Gooley TA, Leisenring W, Crowley J, Storer BE: Estimation of failure probabilities in the presence of competing risks: new representations of old estimators. Stat Med 18:695-706, 1999

3. Higuchi Y, Serizawa T, Nagano O, Matsuda S, Ono J, Sato M, et al: Three-staged stereotactic radiotherapy without whole brain irradiation for large metastatic brain tumors. Int J Radiat Oncol Biol Phys 74:1543-1548, 2009

4. Kanda Y: Investigation of the freely available easy-to-use software 'EZR' for medical statistics. Bone Marrow Transplant 48:452-458, 2013

5. Lorenzoni J, Devriendt D, Massager N, David P, Ruíz S, Vanderlinden B, et al: Radiosurgery for treatment of brain metastases: estimation of patient eligibility using three stratification systems. Int J Radiat Oncol Biol Phys 60:218-224, 2004

6. Pardridge WM: Blood-brain barrier endogenous transporters as therapeutic targets: a new model for small molecule CNS drug discovery. Expert Opin Ther Targets 19:1059-1072, 2015

7. Ruan PK, Gray RJ: Analyses of cumulative incidence functions via non-parametric multiple imputation. Stat Med 27:5709-5724, 2008

8. Serizawa T, Higuchi Y, Nagano O, Hirai T, Ono J, Saeki N, et al: Testing different brain metastasis grading systems in ste- reotactic radiosurgery: Radiation Therapy Oncology Group's RPA, SIR, BSBM, GPA, and modified RPA. J Neurosurg 117 Suppl:31-37, 2012

9. Serizawa T, Higuchi Y, Nagano O, Matsuda S, Ono J, Saeki $\mathrm{N}$, et al: A new grading system focusing on neurological outcomes for brain metastases treated with stereotactic radiosurgery: the modified Basic Score for Brain Metastases. J Neurosurg 121 Suppl:35-43, 2014

10. Serizawa T, Higuchi Y, Ono J, Matsuda S, Nagano O, Iwadate Y, et al: Gamma Knife surgery for metastatic brain tumors without prophylactic whole-brain radiotherapy: results in 1000 consecutive cases. J Neurosurg 105 Suppl:86-90, 2006

11. Serizawa T, Iuchi T, Ono J, Saeki N, Osato K, Odaki M, et al: Gamma Knife treatment for multiple metastatic brain tumors compared with whole-brain radiation therapy. J Neurosurg 93 (Suppl 3):32-36, 2000

12. Serizawa T, Saeki N, Higuchi Y, Ono J, Iuchi T, Nagano O, et al: Gamma Knife surgery for brain metastases: indications for and limitations of a local treatment protocol. Acta Neurochir (Wien) 147:721-726, 2005

13. Sperduto PW, Berkey B, Gaspar LE, Mehta M, Curran W: A new prognostic index and comparison to three other indices for patients with brain metastases: an analysis of 1,960 patients in the RTOG database. Int J Radiat Oncol Biol Phys 70:510-514, 2008

14. Sperduto PW, Kased N, Roberge D, Xu Z, Shanley R, Luo X, et al: Summary report on the graded prognostic assessment: an accurate and facile diagnosis-specific tool to estimate survival for patients with brain metastases. J Clin Oncol 30:419-425, 2012

15. Weltman E, Salvajoli JV, Brandt RA, de Morais Hanriot R, Prisco FE, Cruz JC, et al: Radiosurgery for brain metastases: a score index for predicting prognosis. Int J Radiat Oncol Biol Phys 46:1155-1161, 2000

16. Yamamoto M, Serizawa T, Sato Y, Kawabe T, Higuchi Y, Nagano O, et al: Validity of two recently-proposed prognostic grading indices for lung, gastro-intestinal, breast and renal cell cancer patients with radiosurgically-treated brain metastases. J Neurooncol 111:327-335, 2013

17. Yomo S, Hayashi M, Nicholson C: A prospective pilot study of two-session Gamma Knife surgery for large metastatic brain tumors. J Neurooncol 109:159-165, 2012

\section{Disclosures}

The authors report no conflict of interest concerning the materials or methods used in this study or the findings specified in this paper.

\section{Author Contributions}

Conception and design: Serizawa, Higuchi, Shibamoto. Acquisition of data: Serizawa, Nagano, Aoyagi, Matsuda. Analysis and interpretation of data: Serizawa, Higuchi, Nagano, Aoyagi, Matsuda. Drafting the article: Serizawa. Critically revising the article: Serizawa, Higuchi, Shibamoto. Reviewed submitted version of manuscript: all authors. Approved the final version of the manuscript on behalf of all authors: Serizawa. Statistical analysis: Serizawa, Higuchi, Takemoto. Administrative/technical/material support: Serizawa, Higuchi. Study supervision: Serizawa, Saeki, Shibamoto.

\section{Correspondence}

Toru Serizawa, Tokyo Gamma Unit Center, Tsukiji Neurological Clinic, 1-9-9 Tsukiji, Chuou-ku, Tokyo 104-0045, Japan. email: gamma-knife.serizawa@nifty.com. 